\title{
Adverse Reactions to Titanium Surgical Staples in a Patient After Cholecystectomy
}

\author{
Frederick Tiesenga, MD, FACS, Jenny Wang, MD, Christina Crews, APN NP-C \\ West Suburban Hospital, Westlake Hospital, Presence Health Systems, Elmhurst Hospital, Elmwood Park, IL (all authors).
}

Citation Tiesenga F, Wang J, Crews C. Adverse reactions to titanium surgical staples in a patient after cholecystectomy. CRSLS e2014.03056. DOI 10.4293/CRSLS.2014.03056.

Copyright (C) 2014 SLS This is an open-access article distributed under the terms of the Creative Commons Attribution-Noncommercial-ShareAlike 3.0 Unported license, which permits unrestricted noncommercial use, distribution, and reproduction in any medium, provided the original author and source are credited.

Address correspondence to: Christina Crews, APN NP-C, Ticsenga Surgical Associates, 1950 N Harlem Ave, Elmwood Park, IL Leonon, Telephone: 773-934-9827.

E-mail: christina@newhopesurgical.com

\section{INTRODUCTION}

Titanium is a metal known for its biocompatibility and corrosion resistance. ${ }^{1,2}$ Its uses in the medical field range from long-term orthopedic implants and pacemakers to daily-use articles such as eyeglass frames and ornamental body piercings. ${ }^{3}$ Metal allergies are classically known to occur with nickel, gold, cobalt, and chrome ${ }^{3}$. Titanium has not been fully recognized as an allergen; however, there have been a number of documented incidents of patients with a possible sensitivity to titanium. ${ }^{4-10}$ One specific case reported by Tamai et al. identifies surgical metal clips as the source of an allergen for a breast cancer patient who underwent breast-conserving therapy. The patient, who had a known history of atopic dermatitis and many allergies to foods and drugs, developed worsening atopic dermatitis that was ultimately caused by titanium surgical clips. $^{6}$ We report here another case of possible allergic reaction to titanium surgical clips used in a patient for a cholecystectomy procedure.

\section{CASE PRESENTATION}

A 61-year-old woman presented to the clinic with a request to remove the titanium staples that had been placed from the cholecystectomy she had undergone 10 months prior. The patient had complaints of nonspecific symptoms such as a burning sensation in her left arm, conjunctivitis, irregular bowel movements, and sinusitis with postnasal drip in the morning, all of which began after her cholecystectomy. She had a history of localized allergic reactions to titanium plates from a prior ankle surgery, after which she experienced poor wound healing, erythema, and edema to her leg soon after. All of those resolved after removal of the plates. The patient said that her titanium allergy was disclosed to the surgeon before surgery, but the titanium staples were still used despite this knowledge. Soon after the surgery, the symptoms described above began to develop. Review of medical records showed that her memory lymphocyte immunostimulation assay (MELISA) had equivocal results for titanium allergy. Because she had these symptoms of discomfort and a history of adverse reactions to titanium in addition to the equivocal results, it was decided to proceed with the removal surgery. A radiological study of her abdomen revealed 9 titanium clips in the right upper quadrant of her abdomen, which were removed laparoscopically.

\section{RESULTS}

The patient tolerated the procedure well. On postoperative day 1 , she reported diminished conjunctival swelling. Three days postoperatively, she reported a decrease in postnasal drip and sinus congestion, and after 1 week, her sinusitis had resolved completely. In addition, during her 1-week postoperative visit, she described resolution of the burning sensation to her left arm as well as regular and formed bowel movements. After 1 month postsurgery, she reported being symptom free.

\section{DISCUSSION}

The symptoms of titanium allergy have been reported to manifest in a number of different ways: eczema and erythema over a pacemaker's implantation site, ${ }^{4}$ failed orthopedic implants with poor fracture healing and localized eczema, ${ }^{5,7}$ skin irritation around percutaneous implants for hearing aids, ${ }^{8}$ and gingival hyperplasia from intraoral titanium implants 9 A few studies have speculated that the allergies could be caused by impurities of the titanium materials rather than the titanium itself. ${ }^{10,11}$ Another hy- 
pothesis suggests that titanium implants may corrode and release ions that initiate the allergic response; however, Huang et al. found that titanium released very few ions in $\mathrm{pH} \geq 3.75 .^{12}$ Another study investigated the high affinity of titanium to proteins; titanium-bound cell membrane proteins may induce autoimmune reactions, and titaniumbound intracellular proteins may disrupt normal cell physiology. ${ }^{13}$ Finally, another study conjectures that titanium can activate macrophages, either directly or subsequent to phagocytosis. Activated macrophages can then secrete both pro- and anti-inflammatory cytokines. ${ }^{14}$

Diagnosing titanium allergy can be difficult. Among these different cases, there has not been a standardized valid patch test preparation for titanium allergy testing. Okamura et al. suggest that $0.1 \%$ and $0.2 \%$ titanium sulfate and $0.1 \%$ and $0.2 \%$ titanium chloride solutions were successful reagents for titanium skin-patch testing. ${ }^{15}$ Other options are lymphocyte transformation tests or the MELISA, which may be a more sensitive tests than patch tests. ${ }^{16}$ Results from these tests in combination with the clinical symptoms can warrant removal of the offending material from the patient to resolve symptoms.

The symptoms experienced by the patient in this case are different from that of previously reported cases, and some may not be completely attributed to titanium sensitivity. However, the patient had an equivocal MELISA test result, and with her history of reaction to titanium ankle plates, it was decided to proceed with the removal of the staples. The patient had relief of a majority of her symptoms after her surgery, which led us to believe that some of her discomfort was caused by titanium allergy.

\section{CONCLUSION}

Titanium allergy testing is still being investigated by the medical community. It is worth the consideration to test for this allergy and other metals if the patient presents with irritation after being exposed to titanium. Removal or replacement of the titanium implant would then be appropriate treatment.

\section{References:}

1. Williams DF. Titanium as a metal for implantation. Part 1: physical properties. J Med Eng Technol. 1977;1:195-198, 202.

2. Williams DF. Titanium as ametal for implantation. Part 2: biological properties and clinical applications. J Med Eng Technol. 1977;1:266-270.
3. Forte G, Petrucci F, Bocca B. Metal allergens of growing significance: epidemiology, immunotoxicology, strategies for testing and prevention. Inflamm Allergy Drug Targets. 2008;7:145-162.

4. Yamauchi R, Morita A, Tsuji T. Pacemaker dermatitis from titanium. Contact Derm. 2000;42:52.

5. Thomas P, Bandl WD, Maier S, Summer B, Przybilla B. Hypersensitivity to titanium osteosynthesis with impaired fracture healing, eczema, and T-cell hyperresponsiveness in vitro: case report and review of the literature. Contact Derm. 2006;55:199-202.

6. Tamai K, Mitsumori M, Fujishiro S, et al. A case of allergic reaction to surgical metal clips inserted for post-operative boost irradiation in a patient undergoing breast-conserving therapy. Breast Cancer. 2001;8(1):90-92.

7. Lalor PA, Revell P, Gray AB, Wright S, Railton GT, Freeman MA. Sensitivity to titanium. A cause of implant failure? J Bone Joint Surg Br. 1991;73(1):25-28.

8. Holgers KM, Thomsen P, Tjellstrom A. Persistent irritation of the soft tissue around an osseointegrated titainium implant. Case report. Scand J Reconstr Surg Hand Surg. 1994;28(3):255-230.

9. Mitchell L, Synnott SA, VanDercreek JA. Tissue reaction involving an intraoral skin graft and CP titanium abutments: a clinical report. Int J Oral Maxillofac Implants. 1990;5(1):79-84.

10. Harloff T, Honle W, Holzwarth U, Bader R, Thomas P, Schuh A. Titanium allergy or not? "Impurity" of titanium implant materials. Health. 2010;2(4):306-310.

11. Bircher AJ, Stern WB. Allergic contact dermatitis from "titanium" spectacle frames. Contact Derm. 2001;45:244-245.

12. Huang $\mathrm{HH}$, Chiu $\mathrm{YH}$, Lee $\mathrm{TH}$, et al. Ion release from NiTi orthodontim wires in artificial saliva with various acidities. Biomaterials. 2003;24(20):3585-3592.

13. Stejskal VD, Hudecek R, Stejskal J, Sterzl I. Diagnosis and treatment of metal-induced side-effects. Neuro Endocrinol Lett. 2006;27(suppl 1):7-16.

14. Nakashima Y, Sun DH, Trindade M, Maloney W, Goodman $\mathrm{S}$, Schurman D. Induction of macrophage C-C chemokine expression by titanium alloy and bone cement particles. $J$ Bone Joint Surg. 1999;81(1):155-162.

15. Okamura T, Morimoto M, Fukushima D, Yamane G. A skin patch test for the diagnosis of titanium allergy (abstract at the Japanese Association for Dental Research 46th annual meeting). J Dent Res. 1999;78:1135.

16. Valentine-Thon E, Schiwara HW. Validity of MELISA for metal sensitivity testing. Neuroendocrinol Lett. 2003;24(1-2):57-64. 